\title{
Interferometric measurement of the resonant absorption and refractive index in rubidium gas
}

\author{
K. G. Libbrecht ${ }^{\mathrm{a})}$ and M. W. Libbrecht \\ Department of Physics, California Institute of Technology, Pasadena, California 91125
}

(Received 17 April 2006; accepted 14 July 2006)

\begin{abstract}
We present a laboratory demonstration of the Kramers-Kronig relation between the resonant absorption and refractive index in rubidium gas. Our experiment uses a rubidium vapor cell in one arm of a simple Mach-Zehnder interferometer. As the laser frequency is scanned over an atomic resonance, the interferometer output is affected by variations of both the absorption and refractive index of the gas with frequency, all of which can be calculated in a straightforward manner. Changing the vapor density and interferometer phase produces a family of different output signals. The experiment was performed using a commercially available tunable diode laser system that was designed specifically for the undergraduate physics laboratory. As a teaching tool this experiment is reliable, fun, and instructive, while it also introduces the student to some sophisticated and fundamental physical concepts. (C) 2006 American Association of Physics Teachers.

[DOI: $10.1119 / 1.2335476]$
\end{abstract}

\section{INTRODUCTION}

Tunable lasers are useful tools for teaching atomic and optical physics, because they provide a bright source of variable-frequency, monochromatic light for probing atomic transitions. The advent of tunable diode lasers has made it economically feasible to bring these tools into the undergraduate laboratory, ${ }^{1-4}$ and TeachSpin ${ }^{5}$ has recently introduced a laser system designed specifically for physics teaching. Saturated-absorption spectroscopy is the standard undergraduate lab experiment using tunable diode lasers, but many others are possible. To fully exploit these laser systems, the instructor needs a range of experiments that can be done without the need for much additional (usually expensive) optical hardware. We describe one such experiment that explores the optical properties, both absorption and refractive index, of an atomic gas at optical frequencies near an atomic transition. It uses essentially the same hardware as saturated-absorption spectroscopy, yet it demonstrates much different physics. Furthermore, our experiment is more quantitative than saturated-absorption spectroscopy in that the observed spectra can be accurately calculated in advance.

The underlying physics of this experiment is that the absorption and refractive index in an atomic gas are related via the Kramers-Kronig relation, which is described in several popular textbooks. ${ }^{6,7}$ Optical absorption near an atomic resonance is straightforward to observe by measuring the light transmitted through a gas cell as a function of laser frequency. Measuring changes in the index of refraction of a vapor can be more challenging, because even a sizable total absorption produces an optical phase lag of only a few radians.

We approached this problem by placing a vapor cell in one arm of a Mach-Zehnder interferometer and scanning the laser frequency over the atomic resonance. Both the absorption and index variations contribute to the overall interferometer signal, which we model to compare with experiment.

We have found that all three aspects of this experimentunderstanding the optical properties of an atomic gas, modeling the rather complex interferometer signal, and setting up the hardware-have significant pedagogical value. All three aspects are challenging, but can be readily accomplished with some effort. The remainder of this paper describes the theory and experiment in detail.

\section{A CLASSICAL ATOMIC MODEL}

The first step in this experiment is to calculate the relation between the resonant absorption and the refractive index in a rubidium vapor and to apply it to our laboratory interferometer. We examine this relation only for the specific case of an atomic gas, which is simpler to derive than the general Kramers-Kronig relation, making the physics easier to comprehend.

We begin by considering atoms at rest in the laboratory frame. We use a simple, semi-classical model of a rubidium atom, namely, that of a single electron bound by a harmonic force, acted upon by the electric field of an incident laser. Although crude, this model allows us to derive the basic optical properties of a gas of atoms near an atomic resonance. In this picture, the equation of motion for the electron around the atom is

$$
m\left[\ddot{x}+\gamma \dot{x}+\omega_{0}^{2} x\right]=-e E(x, t),
$$

where $x$ is the position of the electron along the electric field direction, $m$ is the electron mass (we assume the nucleus has effectively infinite mass compared to the electron), $e$ is the magnitude of the electron charge, $\gamma$ is a phenomenological damping term, and $\omega_{0}$ is the usual resonant frequency of the simple harmonic motion. If the electric field varies in time as $E e^{-i \omega t}$, then the dipole moment contributed by a single atom is

$$
p=-e x=\left(e^{2} / m\right)\left(\omega_{0}^{2}-\omega^{2}-i \omega \gamma\right)^{-1} E=\epsilon_{0} \chi_{e} E,
$$

where $\chi_{e}$ is the electric susceptibility. If there are $N$ atoms per unit volume, then the (complex) dielectric constant of the gas is given by

$$
\epsilon(\omega) / \epsilon_{0}=1+4 \pi \chi_{e}=1+\frac{4 \pi N f e^{2} / m}{\left(\omega_{0}^{2}-\omega^{2}-i \omega \gamma\right)},
$$

where $f$ is the oscillator strength of the transition. The oscillator strength is of order unity for strong transitions such as the $S \rightarrow P$ rubidium lines, and is much smaller for forbidden 


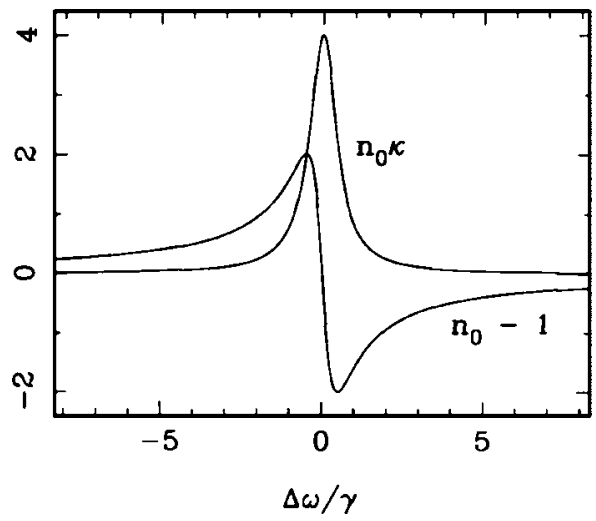

Fig. 1. Plot of the absorption $n_{0} \kappa$ and refractive index change $n_{0}-1$ for a gas near an atomic resonance (arbitrary units).

atomic transitions. Both the oscillator strength and the damping factor $\gamma$ are difficult to calculate for multielectron atoms, which requires a considerable amount of detailed atomic physics.

Maxwell's equations for a propagating electromagnetic wave give

$$
\nabla^{2} E-\mu \epsilon \frac{\partial^{2} E}{\partial t^{2}}=0 .
$$

We define an index of refraction $n=c / v=\sqrt{\epsilon \mu / \epsilon_{0} \mu_{0}}$, where $v$ is the speed of wave propagation. If we assume $\mu / \mu_{0} \simeq 1$ and Eq. (3) for the dielectric constant $\epsilon / \epsilon_{0}$, we find a complex index of refraction that we write as

$$
n=\sqrt{\epsilon / \epsilon_{0}}=n_{0}(1+i \kappa),
$$

where $n_{0}$ and $\kappa$ are real (frequency-dependent) quantities. Equation (5) in the limit of low atomic density gives

$$
n(\omega) \simeq 1-\frac{\pi N f e^{2} \Delta \omega / \omega_{0} m}{\Delta \omega^{2}+\gamma^{2} / 4}+\frac{i \pi N f \gamma e^{2} / 2 \omega_{0} m}{\Delta \omega^{2}+\gamma^{2} / 4} .
$$

Hence,

$$
\begin{aligned}
& \operatorname{Re}(n)=n_{0} \simeq 1-\frac{\pi \Delta \omega N f e^{2} / m \omega_{0}}{\Delta \omega^{2}+\Gamma^{2}} \\
& \operatorname{Im}(n)=n_{0} \kappa \simeq \frac{\pi N \Gamma f e^{2} / m \omega_{0}}{\Delta \omega^{2}+\Gamma^{2}},
\end{aligned}
$$

where $\Delta \omega=\omega-\omega_{0}$ and $\Gamma=\gamma / 2$. These functions are plotted in Fig. 1. Note that far from resonance the index of refraction increases with increasing frequency, which is called normal dispersion. Near resonance the index decreases with $\omega$, which is called anomalous dispersion. (It is helpful to point out this behavior to students, as it helps them avoid sign errors when doing the calculation.)

Note also that there exists a relation between the index of refraction and the absorption of the gas

$$
n_{0}-1 \simeq-2 \Delta \omega n_{0} \kappa / \gamma \simeq-\Delta \omega n_{0} \kappa / \Gamma,
$$

which is independent of the vapor density and oscillator strength of the atomic transition. This relation, showing that $n_{0}(\nu)$ and $\kappa(\nu)$ can be derived from one another, is an example of the more general Kramers-Kronig relation. A full quantum mechanical treatment also yields the same relation for the absorption and refractive index of a gas near an

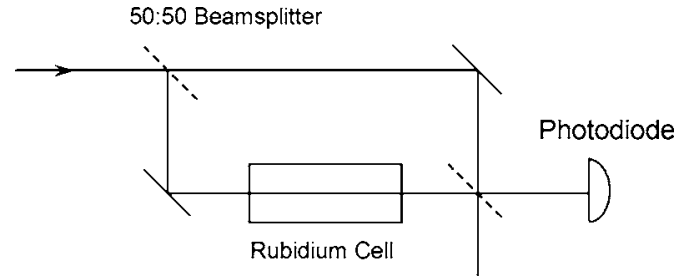

Fig. 2. The basic experimental setup, consisting of a rubidium vapor cell in one arm of a Mach-Zehnder interferometer. The dotted lines represent 50:50 beamsplitters. The input laser scans across a (Doppler broadened) rubidium absorption line.

atomic resonance. Our goal here is not to explore the mathematical subtleties of the Kramers-Kronig relation, but rather to describe a simple experiment that allows the student to observe both the absorption and index of refraction variations of rubidium gas around the $S \rightarrow P$ resonance lines.

\section{AN INTERFEROMETER EXPERIMENT}

We use the experimental setup shown schematically in Fig. 2, which consists of a rubidium vapor cell in one arm of a Mach-Zehnder interferometer. ${ }^{8}$ The input laser light is first split by a beamsplitter (we will assume both beamsplitters in the interferometer are lossless 50:50 beamsplitters), and the two beams travel down different paths through the interferometer. They are recombined at the second beamsplitter, and the light intensity in one direction is measured with a photodetector. The intensity seen at the photodiode is sensitive to both the amplitudes and the relative phases of the two beams as they interfere at the second beamsplitter. In our experiment the photodiode output is recorded as the laser is scanned across a rubidium absorption line.

For a linearly polarized electromagnetic wave propagating through an atomic vapor (with the atoms at rest), we can write the electric field as

$$
E(z, t)=E_{0} e^{-i(\omega t-n k z)}=E_{0} e^{-k n_{0} \kappa z} e^{-i\left[\omega t-k n_{0} z\right]},
$$

where $k=\omega / c$. In the absence of the rubidium cell it is straightforward to show that the output power hitting the photodiode is given by

$$
\frac{I}{I_{0}}=\frac{1}{4}\left|e^{i k L_{1}}+e^{i k L_{2}}\right|^{2}=[1+\cos (k \Delta L)] / 2,
$$

where $L_{1}$ and $L_{2}$ are the lengths of the two separate paths through the interferometer and $\Delta L=L_{2}-L_{1}$. The argument of the cosine can be written as $k \Delta L=\left(k_{0}+\Delta k\right) \Delta L$, where $k_{0}$ $=\omega_{0} / c$ and $\Delta k=\Delta \omega / c$ is the change in $k$ as the laser frequency is scanned over the rubidium resonance. We can make the simplifying approximation that $k \Delta L \simeq k_{0} \Delta L$ as long as $\Delta k \Delta L \ll 1$. In practice, we will scan the laser over the Doppler-broadened rubidium absorption feature, so $\Delta k$ $\lesssim(2 \pi / c) \times(2 \mathrm{GHz})$. In this case the approximation holds as long as $\Delta L \ll 1.5 \mathrm{~cm}$, which is fairly easy to accomplish when setting up the interferometer.

With this equal-path-length approximation we have 


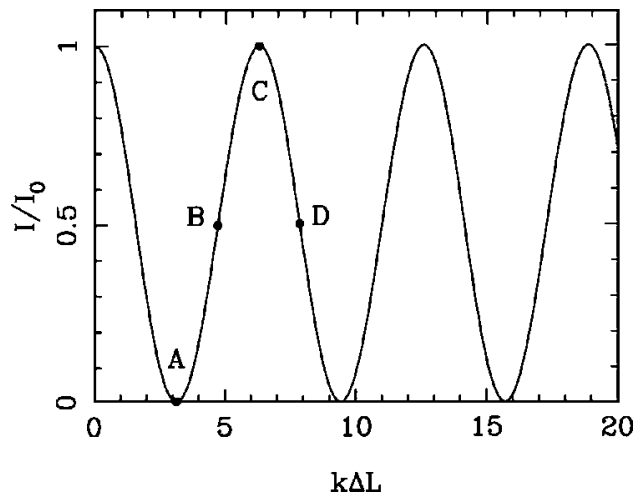

Fig. 3. Photodiode output versus $k \Delta L$, where $k=\omega / c=2 \pi / \lambda$ for a perfect Mach-Zehnder interferometer with no rubidium cell at fixed laser frequency.

$$
\frac{I}{I_{0}} \simeq\left[1+\cos \left(k_{0} \Delta L\right)\right] / 2,
$$

which is plotted in Fig. 3. For future reference, we have labeled points A through D in the phase of the interferometer in Fig. 3.

Next consider the effect of the rubidium cell on the propagation of a laser. From Eq. (10) the total phase shift and absorption upon passing through the cell is

$$
e^{-k n_{0} \kappa \Delta z} e^{i k n_{0} \Delta z}=e^{-k n_{0} \kappa \Delta z} e^{i k \Delta z} e^{i k\left(n_{0}-1\right) \Delta z}=e^{-\tau} e^{i k \Delta z} e^{i \delta},
$$

where $\Delta z$ is the length of the cell. The factor $e^{i k \Delta z}$ in this expression is the free-space propagation factor. The $e^{-\tau}$ factor comes from attenuation in the cell, with $\tau=k n_{0} \kappa \Delta z$.

In the absence of Doppler broadening, Eq. (8) becomes

$$
\tau(\omega)=\frac{\tau_{0} \Gamma^{2}}{\Delta \omega^{2}+\Gamma^{2}},
$$

where $\tau_{0}$ is the absorption at the line center. The $e^{i \delta}$ factor is the additional phase shift from the refractive index of the rubidium atoms, with

$$
\delta(\omega)=k\left(n_{0}-1\right) \Delta z=-\frac{\tau_{0} \Gamma \Delta \omega}{\Delta \omega^{2}+\Gamma^{2}} .
$$

Thus with the rubidium cell in the interferometer the photodiode output will be given by

$$
\begin{aligned}
\frac{I}{I_{0}} & =\frac{1}{4}\left|e^{i k L_{1}}+e^{-\tau} e^{i k L_{2}} e^{i \delta}\right|^{2} \\
& =\left[1+e^{-2 \tau}+2 e^{-\tau} \cos \left(k_{0} \Delta L+\delta\right)\right] / 4,
\end{aligned}
$$

where $\tau$ and $\delta$ are given by Eqs. (14) and (15) (in the absence of Doppler broadening). Note that if the rubidium density is zero, then $\tau=\delta=0$. and we have the same result as before.

In our teaching lab, we stop the calculation here and have the students plot $I / I_{0}$, which is equal to the photodiode signal as a function of $\Delta \omega$ as one scans over an atomic resonance, where they use different values for the interferometer phase $k_{0} \Delta L$ and different values for the absorption optical depth $\tau_{0}$. The resulting family of plots are in qualitative agreement with experimental spectra if we assume a linewidth $\Gamma_{\text {eff }}$ that is roughly equal to the Doppler width of the atoms.

\section{INCLUDING DOPPLER BROADENING}

To obtain better agreement between experiment and theory we must include the effects of Doppler broadening. We begin by assuming a Gaussian distribution for the atomic velocities along the $z$ (laser beam) direction

$$
P(v) d v=\frac{1}{\sqrt{\pi} v_{0}} e^{-v^{2} / v_{0}^{2}} d v,
$$

where

$$
v_{0}=\sqrt{\frac{2 k_{B} T}{m}} .
$$

It is useful to incorporate the Doppler shift immediately and write the distribution function in terms of an angular frequency shift $\omega$

$$
P(\Omega) d \Omega=\frac{1}{\sqrt{\pi} \Omega_{0}} e^{-\Omega^{2} / \Omega_{0}^{2}} d \Omega,
$$

where

$$
\Omega_{0}=\frac{2 \pi}{\lambda_{0}} \sqrt{\frac{2 k_{B} T}{m}} .
$$

For the rubidium transition at $780 \mathrm{~nm}$ (ignoring the mass difference between isotopes) Eq. (20) yields

$$
\Omega_{0} / 2 \pi=0.31\left(\frac{T}{300 \mathrm{~K}}\right)^{1 / 2} \mathrm{GHz} .
$$

With this distribution function we can write the Dopplerbroadened absorption as

$$
\tau_{D}(\Delta \omega)=\int_{-\infty}^{\infty} P(\Omega) \frac{\tau_{0} \Gamma^{2}}{(\Delta \omega+\Omega)^{2}+\Gamma^{2}} d \Omega .
$$

The Lorentzian factor in the integrand is much more sharply peaked than the Gaussian $\left(\Gamma \ll \Omega_{0}\right)$, so we can write $P(\Omega)$ $\simeq P(-\Delta \omega)=P(\Delta \omega)$ and take it out of the integral, giving

$$
\begin{aligned}
\tau_{D}(\Delta \omega) & \simeq P(\Delta \omega) \int_{-\infty}^{\infty} \frac{\tau_{0} \Gamma^{2}}{(\Delta \omega+\Omega)^{2}+\Gamma^{2}} d \Omega \\
& \simeq P(\Delta \omega) \pi \tau_{0} \Gamma \simeq \tau_{D 0} g\left(\Delta \omega / \Omega_{0}\right),
\end{aligned}
$$

where $\tau_{D 0}=\tau_{0} \sqrt{\pi} \Gamma / \Omega_{0}$ and $g(x) \simeq e^{-x^{2}}$.

For the $\delta$ integral we have

$$
\begin{aligned}
\delta_{D}(\Delta \omega) & =-\int_{-\infty}^{\infty} P(\Omega) \frac{(\Delta \omega+\Omega) \Gamma \tau_{0}}{(\Delta \omega+\Omega)^{2}+\Gamma^{2}} d \Omega \\
& =-\frac{\xi \tau_{0}}{\sqrt{\pi}} \int_{-\infty}^{\infty} e^{-\left(y-\Delta \omega / \Omega_{0}\right)^{2}} \frac{y}{y^{2}+\xi^{2}} d y \\
& =\tau_{D 0} f_{\xi}\left(\Delta \omega / \Omega_{0}\right),
\end{aligned}
$$

where $\xi=\Gamma / \Omega_{0}$, and

$$
f_{\xi}(x)=-\frac{1}{\pi} \int_{-\infty}^{\infty} e^{-(y-x)^{2}} \frac{y}{y^{2}+\xi^{2}} d y .
$$

Although $f_{\xi}(x)$ is well-behaved for small $\xi$, we have not been able to find an analytical expression for this function in the limit $\xi \rightarrow 0$, so we evaluated the function numerically. 


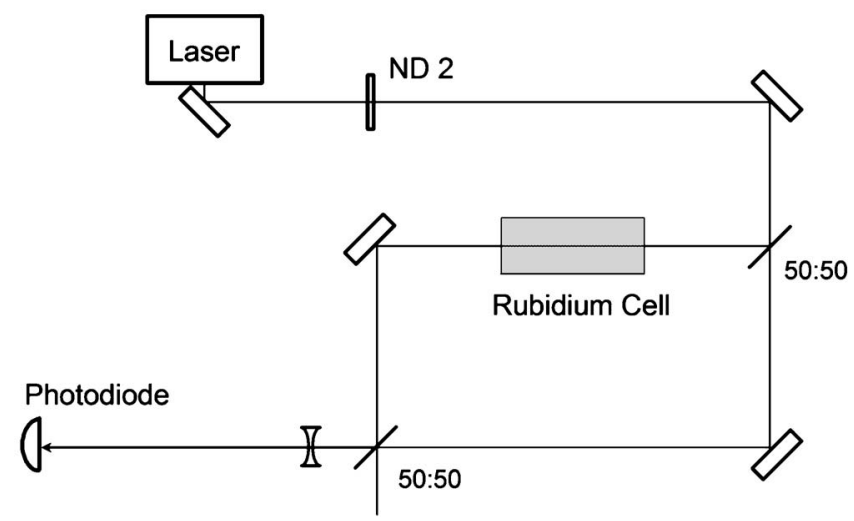

Fig. 4. Optical layout for the experiment. The ND 2 filter has $\approx 1 \%$ transmission and is in place to keep the atomic transition from saturating. The negative lens broadens the beam, making the interference fringes more visible.

We substitute the results for $\tau_{D}(\Delta \omega)$ and $\delta_{D}(\Delta \omega)$, Eqs. (23b) and (24c), respectively, into Eq. (16b) to obtain an expression for the measured interferometer signal $I / I_{0}$, where $I_{0}$ is the off-resonance intensity at maximum brightness [when $\left.\cos \left(k_{0} \Delta L\right)=1\right]$. The calculated signals are discussed in the following along with the experimental results.

\section{THE EXPERIMENT}

The optical setup for this experiment is shown in Fig. 4. The laser was a commercial system ${ }^{5}$ that consists of a temperature-controlled, grating-stabilized, wavelengthtunable diode laser with a linewidth of less than $1 \mathrm{MHz}$. Such a narrow linewidth is not necessary for this experiment because the narrowest spectral features we see are about $50 \mathrm{MHz}$. The rubidium cell, which is part of the commercial system, was $2.5 \mathrm{~cm}$ long and was temperature regulated with a range of $25-100{ }^{\circ} \mathrm{C}$. Precise temperature regulation is not needed for the experiment, but the results are more visually appealing if there is a substantial optical density in the cell. Frequency scans of the laser were typically done at about $10 \mathrm{~Hz}$.

The optics were first set up with the laser far off resonance to obtain high-contrast interferometer fringes while the laser was not being scanned. The negative lens in front of the photodiode increased the size of the beam to make the fringes more visible and ensured that the photodiode does not average over more than a small fraction of a fringe. The fringes were viewed using a video camera while aligning the interferometer. We found it very useful to frequently block and unblock one arm of the interferometer to gauge the overlap of the beams. Once aligned, we tested the quality of the interferometer by gently wiggling one of the folding mirrors with a finger while viewing the photodiode output on an oscilloscope, which yielded traces similar to that shown in Fig. 5. When properly aligned, the fringe contrast defect $I_{\min } / I_{\max }$ is close to zero. We have not fully explored the effect of an intensity imbalance between the two interferometer paths, but we have found it easy to produce satisfactory fringes even with the imbalance caused by the additional reflections from the cell windows. We also took some care to make the two path lengths of the interferometer nearly identical, given the criterion $\Delta L \ll 1.5 \mathrm{~cm}$ described previously.

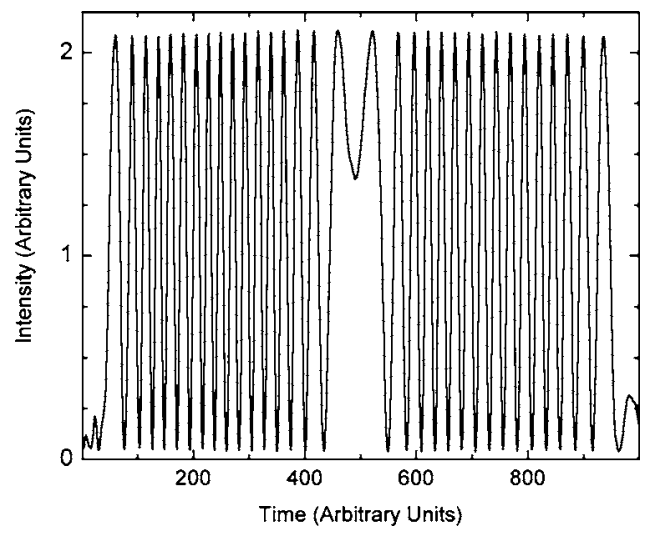

Fig. 5. A single-sweep oscilloscope trace showing the interferometer output as a function of time as a folding mirror was being gently wiggled back and forth. Traces like the one shown here were used to check the alignment of the interferometer and to make sure the fringe contrast defect $I_{\min } / I_{\max }$ was small.

Again we found that path length differences as high as a centimeter or two do not qualitatively alter the interferometer signal.

Blocking one arm of the interferometer while scanning the laser yielded an overall rubidium absorption spectrum similar to that shown in Fig. 6, which shows the transmission through the cell along with a frequency calibration trace as the laser was scanned. Note the absorption filter in Fig. 4, which is needed to prevent the laser from saturating the transition.

The calibration signal in Fig. 6 shows the light transmitted through a confocal Fabry-Pérot cavity with an effective freespectral range $(=c / 4 \ell$, where $\ell$ is the mirror spacing $)$ of $378 \mathrm{MHz}$. We see from this trace that there is a slightly nonlinear relation between the laser scan (the voltage to a piezoelectric stack driving the external feedback grating in the laser head) and the frequency of the output light. For the remainder of the experiment we focused on a $\pm 2.5 \mathrm{GHz}$ scan around the third absorption feature seen in Fig. 6. We used the data in this plot to convert the laser scan to frequency

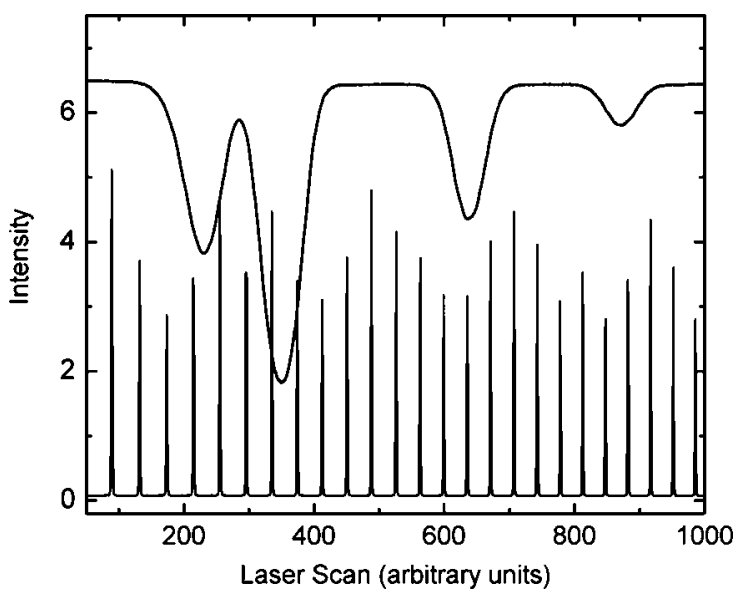

Fig. 6. Top trace: Absorption of a laser beam passing through a rubidium vapor cell, as the laser scans across the rubidium absorption lines near $780 \mathrm{~nm}$. The first and last absorption dips are from ${ }^{87} \mathrm{Rb}$ and the middle two are from ${ }^{85} \mathrm{Rb}$. Bottom trace: Light intensity transmitted through a confocal Fabry-Perot cavity with an effective free-spectral range of $378 \mathrm{MHz}$. 


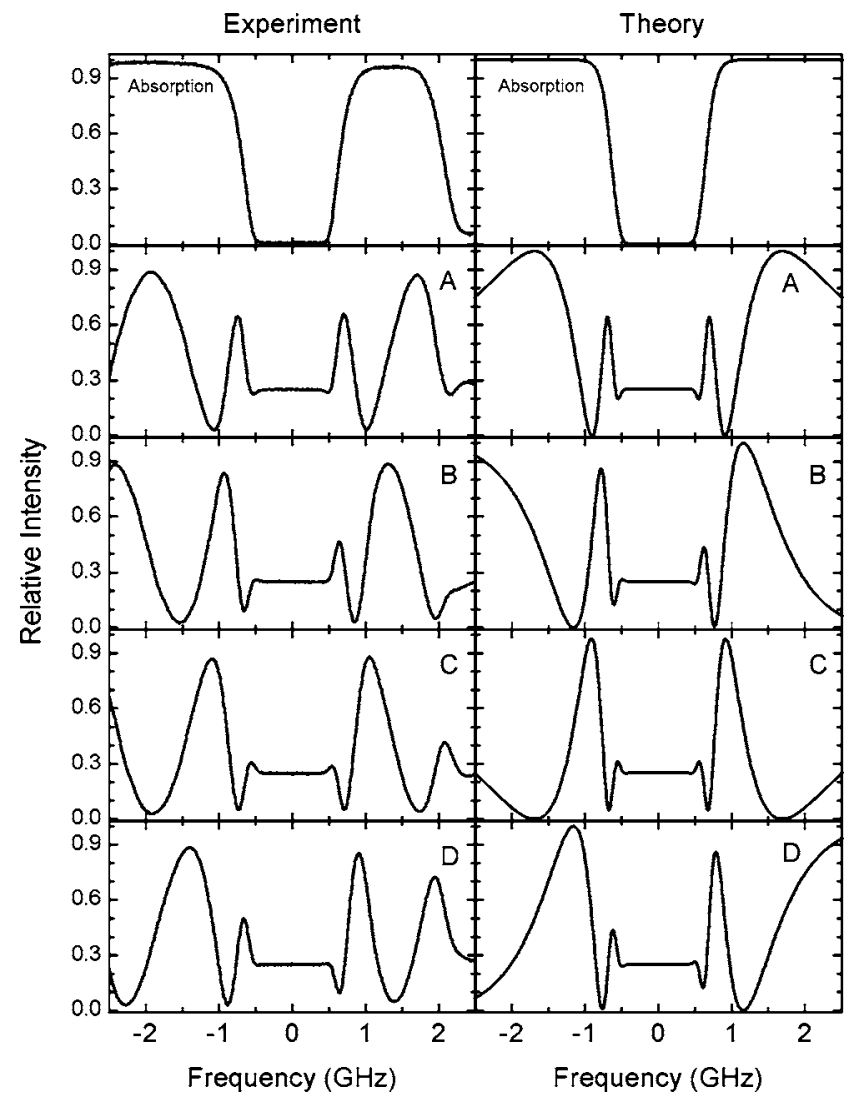

Fig. 7. Interferometer output $I / I_{0}$ as a function of laser frequency and interferometer phase with a rubidium cell temperature of $90^{\circ} \mathrm{C}$. The scan was centered on the third absorption feature seen in Fig. 6. The theory plots were generated using a single value of $\tau_{D 0}=27$, which was adjusted to match the experimental curves. The absorption plot at the top left was taken with one arm of the interferometer blocked. Curves A-D refer to the phase points labeled in Fig. 3. We obtained overall good qualitative agreement between experiment and theory, especially near the line center. At the edges of the scans the data are corrupted by contamination from nearby absorption features (which were not included in the theoretical curves). Note that $I / I_{0}$ goes to 0.25 at the line center, because the beam incident on the rubidium cell is almost completely blocked by absorption.

units, ignoring the slight nonlinearity of the scan. We could also simply use the spacing between the absorption features, which gives sufficient accuracy for comparing with calculations.

We recorded several spectra using the following procedure: (1) Set the rubidium cell temperature and let it stabilize. (2) Block one arm of the interferometer and record a plain rubidium absorption spectrum. (3) Unblock both interferometer arms and record spectra at the four interferometer positions A-D shown in Fig. 3. The phase of the interferometer, $k_{0} \Delta L$, was adjusted by applying a small force to the surface of the optical table, thus bending it slightly and changing $\Delta L$. Theoretical curves were generated by choosing a single value of $\tau_{D 0}$ to give the best qualitative fit to the data for each temperature. Figures 7-9 show comparisons of experimental and theoretical scans at three rubidium cell temperatures.

As can be seen in Figs. 7-9, this demonstration gives good qualitative agreement between theory and experiment. In these plots the measured intensity was normalized to fit the theoretical curves. The absorption and full interferometer

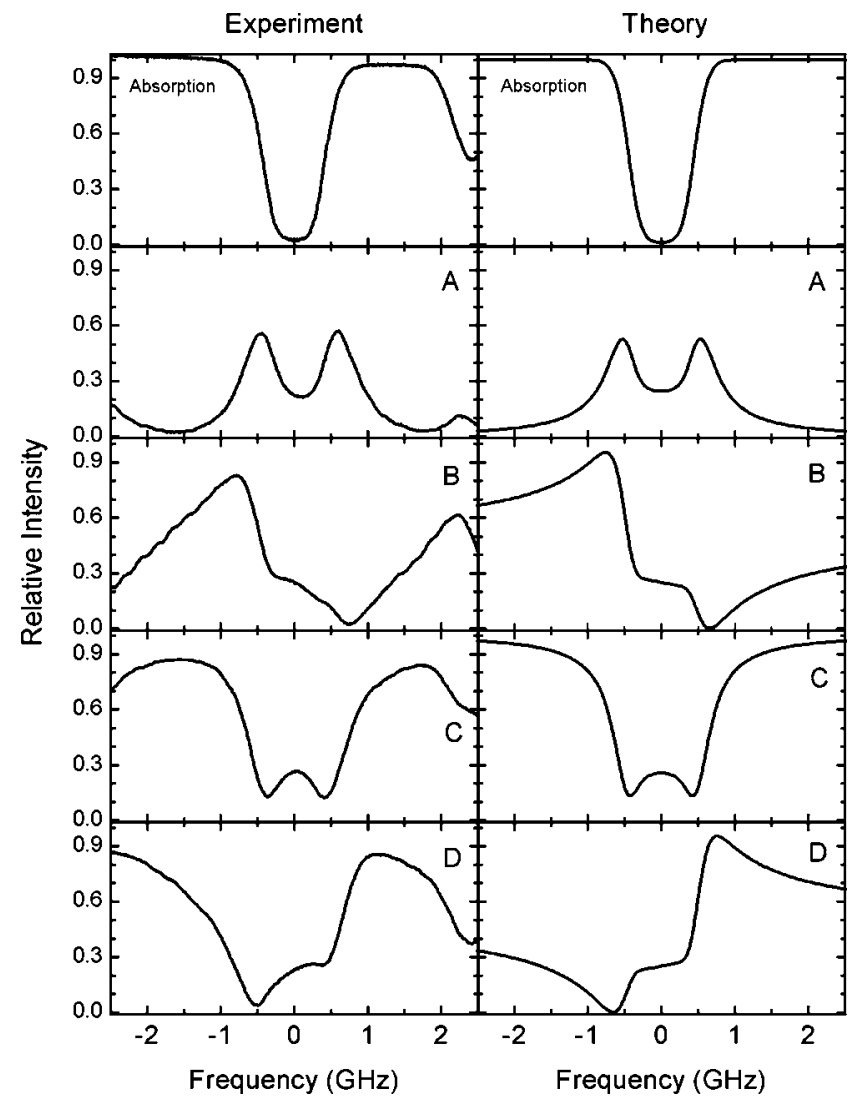

Fig. 8. Same as Fig. 7, but with a rubidium cell temperature of $70^{\circ} \mathrm{C}$ and $\tau_{D 0}=4.5$.

curves were normalized independently, although the same normalization factor was used for curves A-D at each of the three temperatures.

To understand the physics in these plots, consider the case where the rubidium density is high (see Fig. 7). The absorption profile (top panel, where one arm of the intereferometer was blocked) shows that essentially no laser light passes through the cell near resonance, thus yielding a flat-bottomed absorption curve. At higher cell temperatures, the absorption profile broadens further. The experimental plot shows a neighboring absorption feature not seen in the theory plot, which comes from the other rubidium isotope (see Fig. 6), which was not included in the calculation.

When both arms of the interferometer are unblocked (remaining plots in Fig. 7), the relative intensity $I / I_{0}$ goes to 0.25 on resonance when the rubidium density is high, regardless of the phase $k_{0} \Delta L$ of the interferometer. This reduction occurs because there is almost total absorption in the cell on resonance, which has the same effect as blocking that arm of the interferometer. With the cell arm effectively blocked on resonance, the light level is reduced $50 \%$ by the first beamsplitter and another $50 \%$ by the second beamsplitter, yielding a total transmission of 0.25 .

Away from resonance the signal is determined by a complex interplay of absorption and phase shift in the interferometer, which was derived previously and is calculated in detail from Eq. (16a). Note, from comparing Eqs. (14) and (15), that $\tau(\omega) \sim 1 / \Delta \omega^{2}$ and $\delta(\omega) \sim 1 / \Delta \omega$ far from resonance. This dependence means that the phase shift contribution to the interferometer becomes more important than the 


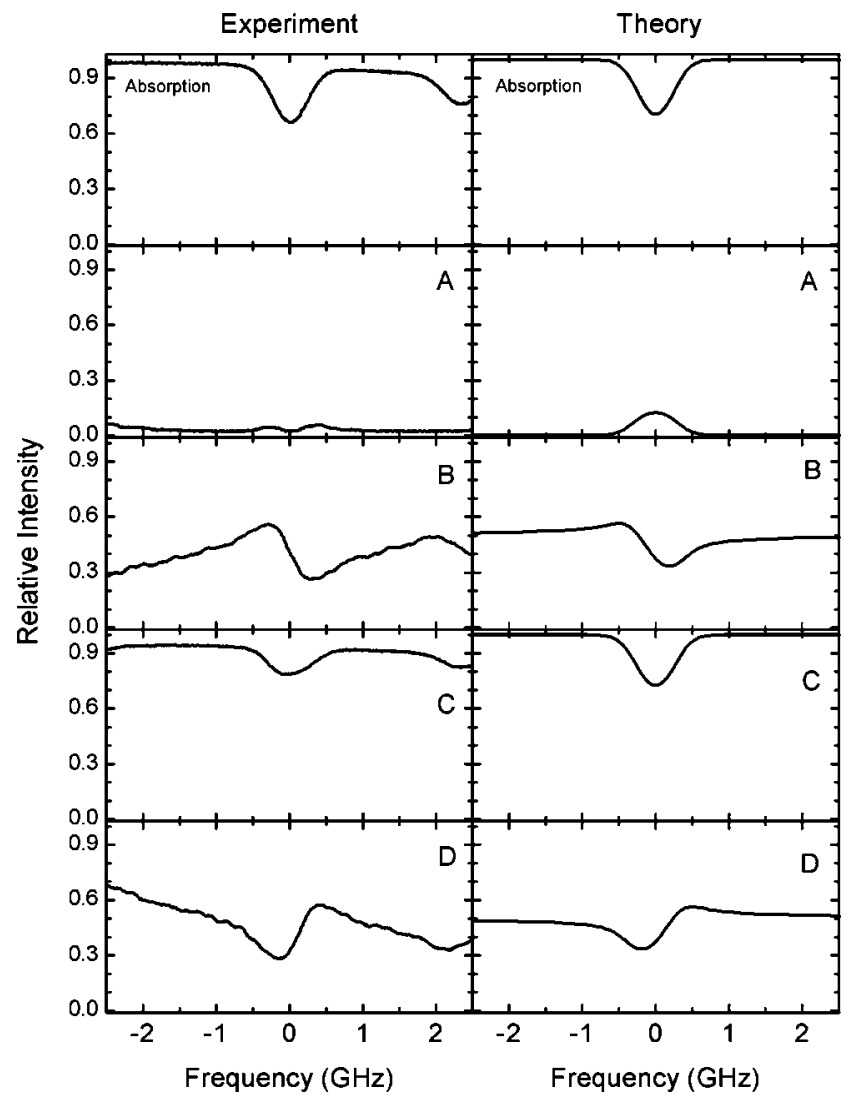

Fig. 9. Same as Fig. 7, but with a rubidium cell temperature of $45^{\circ} \mathrm{C}$ and $\tau_{D 0}=0.35$.

absorption as one looks further from resonance. The phase shift term yields the distinct oscillatory behavior seen in Fig. 7 on either side of the atomic resonance.

The agreement between theory and experiment begins to break down at the edges of our scans, mainly because of contamination from neighboring rubidium resonances. This contamination is most prevalent in the phase $\delta$ at high rubidium densities. Using isotopically pure rubidium would greatly reduce this contamination, but this use would make the experiment more expensive.

Finally, we found that the ratios of the three values of $\tau_{D 0}$ extracted from the data plots were not in good quantitative agreement with what would be expected from the cell temperatures. This discrepancy was because the temperature regulation of our rubidium cell was fairly crude. The temperature was not uniform across the cell, and the measured temperature was not equal to the coldest point on the cell. These limitations could be reduced by better cell design with a more uniform cell temperature.

\section{CONCLUSIONS}

We have been using this experiment in our Advanced Physics Laboratory (for senior physics majors) for several years with good effect. It is straightforward to set up, can be done in an afternoon, and it nearly always works well. Watching the oscilloscope trace while pushing on the table (to change $\Delta L$ in real time) is especially enlightening for students. Qualitatively, the signal is insensitive to misalignment of the interferometer, imbalance in the beams, and other experimental limitations. The Mach-Zehnder interferometer does not reflect light back into the diode (in contrast to a Michelson interferometer), and therefore does not produce unwanted optical feedback effects that can destabilize the laser frequency. The calculation is challenging, but doable, and it teaches many important concepts in optical and atomic physics.

The fact that the student can calculate the interferometer output in some detail before going into the lab is one of the experiment's strong points. It is not necessary to include Doppler broadening to achieve satisfactory qualitative agreement between theory and experiment, and omitting this step simplifies the calculations considerably. The experiment is a good complement to saturated-absorption spectroscopy for teaching interesting physics using tunable diode lasers, and the two experiments can be done using mostly the same equipment.

\section{ACKNOWLEDGMENT}

This material is based upon work supported by the National Science Foundation under Grant No. 0340559.

${ }^{a)}$ Electronic address: kgl@caltech.edu

${ }^{1}$ C. E. Wieman and L. Hollberg, "Using diode-lasers for atomic physics," Rev. Sci. Instrum. 62, 1-20 (1991).

${ }^{2}$ K. B. MacAdam, A. Steinback, and C. Wieman, "A narrow-band tunable diode-laser system with grating feedback, and a saturated absorption spectrometer for Cs and Rb," Am. J. Phys. 60, 1098-1111 (1992).

${ }^{3}$ K. G. Libbrecht, R. A. Boyd, P. A. Willems, T. L. Gustavson, and D. K. Kim, "Teaching physics with $670 \mathrm{~nm}$ diode lasers-construction of stabilized laser and Lithium cells," Am. J. Phys. 63, 729-737 (1995).

${ }^{4}$ R. A. Boyd, J. L. Bliss, and K. G. Libbrecht, "Teaching physics with $670 \mathrm{~nm}$ diode lasers-experiments with Fabry-Perot cavities," Am. J. Phys. 64, 1109-1116 (1996).

${ }^{5}$ TeachSpin, 〈http://www.teachspin.com/ $\rangle$

${ }^{6}$ J. D. Jackson, Classical Electrodynamics (Wiley, New York, 1998), 3rd ed.

${ }^{7}$ A. Yariv, Quantum Electronics (Wiley, New York, 1989), 3rd ed.

${ }^{8}$ Paul Nachman, "Mach-Zehnder interferometer as an instructional tool," Am. J. Phys. 63, 39-43 (1995). 